\title{
EFECTOS DE LA EXTRACCIÓN DE TURBA SOBRE LA COMPOSICIÓN Y ESTRUCTURA DE UNA TURBERA DE SPHAGNUM EXPLOTADA Y ABANDONADA HACE 20 AÑOS, CHILE
}

\author{
EFFECTS OF PEAT EXTRACTION ON THE STRUCTURE AND COMPOSITION OF \\ SPHAGNUM PEATLAND EXPLOITED AND ABANDONED FOR 20 YEARS, CHILE
}

Erwin Domínguez ${ }^{1}$, Nelson Bahamonde ${ }^{2}$ \& Christian Muñoz-Escobar ${ }^{3}$

\begin{abstract}
Magellanic Sphagnum peatlands are characterized by their ability to maintain biodiversity and water cycle of subantarctic ecosystems, together withits contribution to the global carbon storage through the slow production and accumulation of peat. Through industrial peat extraction, these wetlands have become an economic resource for the past 30 years, generating impacts on the landscape, hydrology and flora of these ecosystems. To evaluate the effects of peat extraction on peatland vegetation, we studied a Sphagnum peatland exploited 20 years ago in the locality of Río Rubens, Province of Última Esperanza, Magallanes, Chile. 42 plots of $1 \mathrm{~m}^{2}$ were studied in order to determine differences in species composition and plant coverage. In total 24 taxa were identified, 8 of these were alien to the study area, all classified as invasive. Significant differences where found in floristic composition between the exploited and non exploitedares. The mechanical modification of the area, as a result of peat extraction and construction of drainage channels, favors the arrival of exotic flora specimens such as Rumex acetosella, Carex canescens, Holcus lanatus and Hieracium pilosella.
\end{abstract}

Key words: Flora, Invasive plants, Wetlands, Sphagnum peatland, Peat, Southern Patagonia.

1 Instituto de Investigaciones Agropecuarias - INIA - CRI - Kampenaike. Casilla 277, Punta Arenas, Chile. edominguez@inia. $\mathrm{cl}$

2 Instituto de Investigaciones Agropecuarias - INIA - CRI - Kampenaike. Casilla 277, Punta Arenas, Chile.

3 Programa de Doctorado en Sistemática y Biodiversidad, Facultad de Ciencias Naturales y Oceanográficas, Departamento de Zoología, Universidad de Concepción, Concepción. 


\section{RESUMEN}

Las turberas de Sphagnum de la región de Magallanes se caracterizan por contribuir a mantener la biodiversidad y el ciclo hídrico de los ecosistemas subantárticos, además de aportar al almacenamiento mundial de carbono, a través de la producción y acumulación lenta de materia orgánica en forma de turba. Estos humedales se han convertido en un recurso económico a través de la extracción minera de turba durante los últimos 30 años, generando impactos sobre el paisaje, la hidrología y la flora de estos ecosistemas. Para evaluar los efectos de dicha explotación sobre la vegetación, se estudió una turbera de Sphagnum explotada hace 20 años, en el sector de Río Rubens, Provincia de Última Esperanza, Magallanes, Chile. Con este objetivo se establecieron 42 parcelas de $1 \mathrm{~m}^{2}$ a fin de determinar diferencias en la composición y cobertura de especies. En total se identificaron 24 taxa, 8 de éstas fueron no nativas, exclusivas de la zona intervenida, todas clasificadas como invasoras, mientras que no hubo especies exóticas en la zona no intervenida. Se encontraron diferencias significativas en la composición florística de las áreas intervenidas respecto de las no explotadas. Finalmente, la modificación mecánica como producto de la extracción de turba y la construcción de canales de drenaje, favorece el arribo de especies exóticas de ambientes más secos tales como: Rumex acetosella, Holcus lanatus, Carex canescens y Hieracium pilosella.

Palabras clave: Flora, plantas invasoras, Humedal, Turbera de Sphagnum, Turba, Patagonia austral.

\section{INTRODUCCIÓN}

Las turberas son un tipo de humedal de zonas de clima frío a frío-templado, dominado por plantas hidrófilas, que se caracteriza por producir y acumular materia orgánica proveniente de la descomposición parcial de restos vegetales de variado espesor, que se transforma finalmente en turba dada la ausencia de aire y la presencia de un medio ácido o básico y saturado de agua (Broder et al. 2012, Ramsar, 2004, Roig \& Roig 2004). Las turberas representan entre el $50 \%$ y $70 \%$ de todos los humedales y cubren un equivalente de sólo un 3\% (4 millones de $\mathrm{km}^{2}$ ) de la superficie del planeta (Parish et al. 2008). Se distribuyen principalmente en el hemisferio norte, mientras que sólo un $4 \%$ de las turberas está presente en América del Sur, principalmente en Chile y Argentina (Landry et al. 2010, Díaz et al. 2008). En su conjunto, éstas contienen dos veces el carbono almacenado en todos los bosques del mundo, cantidad comparable a todo el carbono que existe en la atmósfera terrestre (Grootjans et al. 2010). Por otra parte, estos ecosistemas proveen servicios ecosistémicos vitales para las sociedades humanas, a escala local, regional y global, contribuyendo a mantener la biodiversidad, el ciclo hídrico y el almacenamiento mundial de carbono (Grootjans et al. 2010, Joosten \& Cowenberg 2008, Joosten \& Clarke 2002).
En la actualidad, las turberas se encuentran amenazadas mundialmente por diferentes actividades antrópicas que llevan a la degradación o desaparición de estos ecosistemas. Entre éstas cabe mencionar la extracción de turba para la producción de sustratos de alta calidad usada en viveros, cultivo de hortalizas y de plantas ornamentales, adquiriendo gran valor comercial en países como Israel, Japón, Holanda, Alemania y Estados Unidos (IUSS Grupo de trabajo WRB 2007, Rochefort, 2000, Salin, 1996).

Dado que la turba es considerada una importante materia prima para la agroindustria, las turberas de Sphagnum adquieren gran relevancia, debido a que presentan una considerable acumulación de turba que puede alcanzar varios metros de espesor. En la Región de Magallanes estos humedales son muy abundantes, producto de la compleja topografía de la zona, originándose en valles y depresiones que corresponden a cuencas lacustres de origen glaciar colmatadas de sedimentos con poco o nada de drenaje, lo que provoca una constante acumulación de agua en condiciones anaeróbicas, lo que unido a las bajas temperaturas hace que microorganismos que promueven la descomposición vegetal actúen muy lentamente, permitiendo así la acumulación de materia orgánica (Iturraspe 2010, Henríquez 2004, Pisano 1977, Pisano 1983). Las turberas de Sphagnum cubren aproximadamente 7.684.000 hectáreas en Chile, según Pliscoff \& Luebert (2006) 


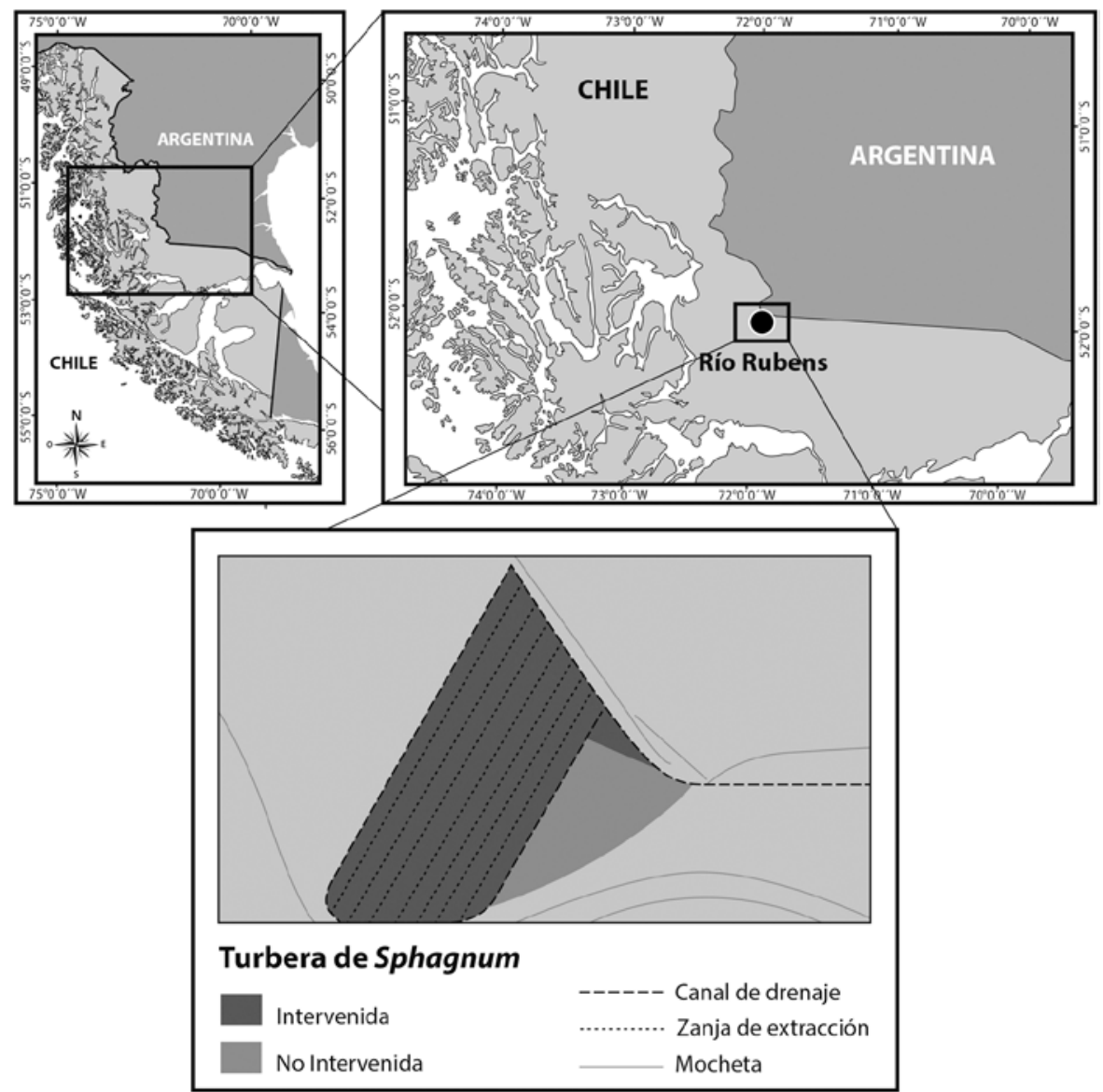

Fig. 1. Mapa de ubicación de sitios de muestreo sector de Río Rubens, Provincia de Última Esperanza, Región de Magallanes

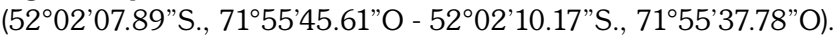

y 1.840.000 en Magallanes según Ruíz \& Doberti (2005), representando el mayor depósito y sumidero de carbono terrestre existente en el hemisferio sur.

En la Región de Magallanes algunas turberas de Sphagnum han sido degradadas por la actividad minera durante los últimos 30 años (Landry et al. 2010), no existiendo hasta ahora planes de mitigación o estudios que analicen el efecto provocado sobre la flora de estos humedales. Se han realizado diversos estudios florísticos en turberas de Sphagnum prístinas o perturbadas por el fuego en la Patagonia y Tierra del Fuego (Pisano, 1977, 1983, Teneb \& Dollenz 2004, Teneb et al. 2008, Díaz et al. 2008, Kleinebecker et al. 2010). Sin embargo, ninguna de estas investigaciones han evaluado los impactos generados por la extracción de la turba en este tipo de humedal, tales como los cambios que ocurren en la etapa de abandono sobre la estructura y composición de la flora, por ejemplo el proceso de invasión biológica, fenómeno que afecta a todos los ecosistemas naturales a escala mundial (Mack et al. 2000), y que requiere de especial atención cuando se trata del establecimiento de especies no nativas. El objetivo de este trabajo es identificar los impactos de la actividad minera sobre la estructura y composición de la flora en una turbera de Sphagnum abandonada, en términos del arribo de especies invasoras como resultado de las actividades de extracción de turba que pueden estar afectando la estructura de estos ecosistemas.

\section{MATERIALES Y MÉTODOS}

En Enero de 2011 se estudió una turbera ubicada en el sector del río Rubens en la Provincia de Última 
Esperanza, Región de Magallanes (5202’07.72”S, $71^{\circ} 55$ '42.71'O) cuya extensión es de aproximadamente 250 ha. Se sitúa en un ambiente en el que se alternan turberas de ciperáceas y unidades más pequeñas de Sphagnum. Un perfil estratigráfico realizado con un barreno colector de muestras (modelo ruso) indica un estrato inferior, más antiguo, conformado por ciperáceas y otro superficial, de 1 a $2 \mathrm{~m}$ de espesor constituido por Sphagnum, lo que demuestra la naturaleza transicional de este ecosistema, adyacente a turberas minerotróficas dominadas por ciperáceas. La turbera fue explotada hace 20 años de manera incompleta, habiéndose extraído aproximadamente 1 $\mathrm{m}$ de espesor de turba en trincheras paralelas, alternadas con fajas no explotadas, además presenta un drenaje perimetral que afecta a toda la turbera (Fig. 1). El sitio de estudio presenta dos condiciones: a) una zona intervenida por acción mecánica, producto de la cosecha de turba con zanjas, mochetas y canales de drenajes y b) una zona sin intervención mecánica, pero con la presencia de un drenaje perimetral. Se realizaron 42 censos de vegetación, utilizando como unidad muestral parcelas de $1 \mathrm{~m}^{2}$, distribuyendo al azar 28 de éstas en el sector intervenido con mayor superficie y 14 parcelas en el sector no intervenido con menor superficie.

La complejidad de la comunidad vegetal presente en cada unidad muestral, se estimó a través de la riqueza de especies (S), cobertura (expresada en porcentaje), diversidad de Shannon (H') (Shannon, 1948) y Gini-Simpson (1- $\left.\lambda^{\prime}\right)$ (Caso \& Gil, 1988). Por cada parámetro comunitario se construyó una matriz de distancia Euclidiana (Legendre \& Legendre, 1983) entre pares de observaciones, con el objeto de realizar test de diferencias entre condiciones de perturbación, utilizando análisis de similitud (ANOSIM) (Clarke \& Green, 1988; Clarke, 1993).

La estructuración del ensamble vegetal se representa y contrasta gráficamente, utilizando un ordenamiento de escalamiento multidimensional no paramétrico (nMDS) (Kruskal, 1964) sobre una matriz de similitud de Bray-Curtis (Bray \& Curtis, 1957, Clarke et al. 2006) la cual utiliza la información de composición de especies con su respectiva cobertura. Se evaluó el efecto de la condición de perturbación, a través de un análisis de similitud (ANOSIM) (Clarke \& Green, 1988) utilizando 50.000 permutaciones con el objeto de estimar su significancia. Por otro lado, se utilizó el índice multivariado de dispersión
(MVDISP) (Warwick \& Clarke, 1993), para medir la variabilidad de las comunidades vegetales entre las muestras en ambas condiciones de estrés producto de la perturbación. Finalmente, para determinar la contribución de las especies en la estructuración de la comunidad de plantas, se realizó un análisis de porcentaje de similitud (SIMPER) (Clarke, 1993), de modo de indicar cuantitativamente qué especies vegetales explican las diferencias entre grupos. Todos los análisis tanto univariados como multivariados se realizaron en el programa PRIMER-E v6.1.12 (Clarke \& Gorley, 20061).

Se identificaron todas las especies presentes en las parcelas, a nivel de género o especie según lo permitiese la muestra. La información taxonómica, origen (nativo, exótico) de las especies registradas fue obtenida de Matthei 1995, Henríquez et al. 1995, Domínguez 2006 y el estatus de invasión según lo propuesto por Pysek et al. 2004.

\section{RESULTADOS Y DISCUSIÓN}

En total se registraron 24 especies, de las cuales 16 son nativas y 8 no nativas. Los taxa corresponden a 17 plantas vasculares, 4 briófitas, 2 líquenes y 1 hongo. El sector cosechado presentó las 8 especies no nativas, todas clasificadas como invasoras, mientras que el área no intervenida sólo presentó especies nativas (Tabla 1). Los resultados, sólo muestran diferencias para la cobertura de especies, siendo ésta significativamente mayor en el sector no intervenido (Tabla 2). Mientras que en términos de riqueza y diversidad $\left(\mathrm{H}^{\prime}, 1-\lambda^{\prime}\right)$ no se observaron diferencias (Tabla 2). Sin embargo, al considerar la composición florística, utilizando una hemimatriz de similitud de Bray-Curtis, sí se obtuvieron diferencias significativas entre los sectores comparados $(R=0,262 ; p<0,001)$, observando una mayor dispersión (MVDISP) en la zona intervenida (1.186) en comparación a la zona no intervenida (0.227) (Fig. 2), dando cuenta de un estrés postimpacto producto del efecto de los canales de drenaje sobre las turberas y modificación en la topografía de la turbera, aumentando así la variabilidad de taxa presentes en estas zonas, siendo la mayoría de estas especies no nativas.

1 Clarke, K.R. \& R.N. Gorley 2006. PRIMER v6: User manual/ Tutorial. PRIMER-E Ltd, Plymouth. 
Tabla 1. Identificación de las especies que caracterizan (\% contribución) la estructuración entre las turberas de Sphagnum intervenidas y no intervenidas, esto según los resultados del análisis SIMPER en el sector de Río Rubens. En gris las especies que explican en al menos un $90 \%$ la formación de los grupos.

Asteraceae

Hieracium pilosella L. ssp. euronotum Nägeli \& Peter

No Nativa

Hypochaeris radicata $L$.

0.28

No Nativa

Caryophyllaceae

Cerastium fontanum Baumg.

0.1

No Nativa

Empetraceae

Empetrum rubrum Vahl ex Willd.

\subsection{8}

\subsection{3}

0.05

Gaultheria pumila (L. f.) D.J. Middleton

Gaultheria antarctica Hook.f.

Nothofagaceae

Nothofagus antarctica (G. Forst.) Oerst.

Polyginaceae

Rumex crispus L.

Rumex acetosella $\mathrm{L}$.

Santalaceae

Nanodea muscosa Banks ex C.F. Gaertn.

Thymelaeaceae

Drapetes muscosus Lam.

1.24

0.3

0.83

0.03

0.48

0.17

0.14

Cyperaceae

Carex canescens $\mathrm{L}$.

Juncus scheuchzerioides Gaudich.

Junco sp.

Tetroncium magellanicum Willd.

Poaceae

Holcus lanatus L.

Agrostis mevenii Trin.

Dicranaceae

Dicranoloma sp.

Polytrichaceae

Polytrichum strictum Brid.

Sphagnaceae

Sphagnum magellanicum Brid

Sphagnum fimbriatum Wilson

Cladoniaceae

Cladonia sp.

Usnaceae

Usnea sp.

Strophariaceae

Galerina patagonica Singer

\subsection{5}

0.11

1.44

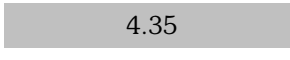

No Nativa

$\begin{array}{ll}6.21 & \text { Nativa } \\ 1.49 & \text { Nativa }\end{array}$

Nativa

Nativa

\begin{tabular}{|l|l|}
\hline 4.87 & No Nativa \\
\hline 0,89 & No Nativa
\end{tabular}

0.37

Nativa

10.41

Nativa

68.96

2.33

Nativa

0.28

Nativa

0.41

1.12

Nativa

0.13

0.47

Nativa

0.1

Nativa 
Tabla 2. Parámetros comunitarios: riqueza (S) y diversidad (H'y 1- $\left.\lambda^{\prime}\right)$ con ANOSIM, previa construcción de una matriz de distancia Euclidiana entre una turbera no intervenida y una intervenida por la extracción de turba.

\begin{tabular}{lllll}
\hline & No Intervenida & Intervenida & $\mathrm{R}$ & $\mathrm{p}$ \\
\hline $\mathrm{S}$ & $5.23 \pm 0.36$ & $4.89 \pm 0.63$ & -0.044 & 0.72 \\
Cobertura & $99.56 \pm 0.15$ & $41.72 \pm 4.64$ & 0.709 & $<0.001$ \\
$\mathrm{H}^{\prime}$ & $0.57 \pm 0.03$ & $0.68 \pm 0.09$ & 0.019 & 0.29 \\
$1-\lambda^{\prime}$ & $0.30 \pm 0.02$ & $0.43 \pm 0.09$ & 0.034 & 0.24 \\
\hline
\end{tabular}

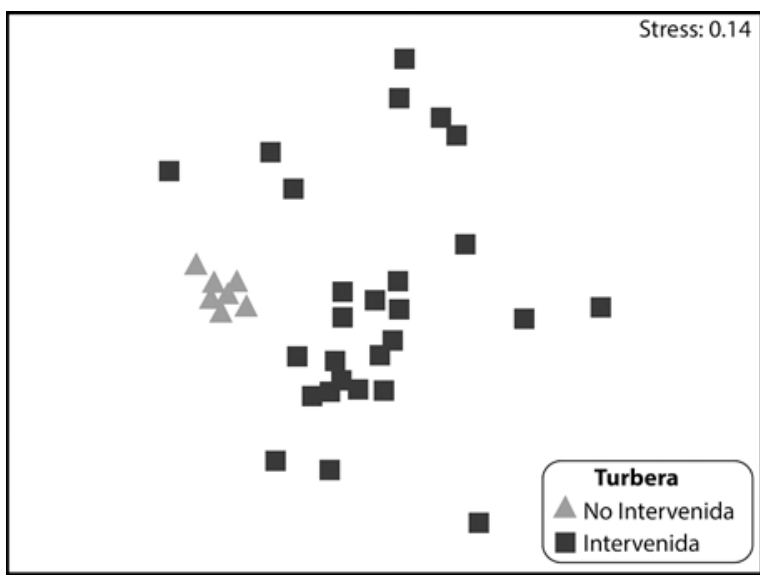

Fig. 2. Ordenación nMDS de las parcelas de muestreo de una turbera de Sphagnum no cosechada (no intervenida) y cosechada (intervenida por la actividad minera), en el sector de Río Rubens.

En relación a las especies que primariamente explican la estructuración de las comunidades de plantas entre sitios, se destacan Sphagnum magellanicum (68,96 \%) y Empetrum rubrum (24,54\%) para el sector no intervenido, siendo estas especies dominantes a lo largo de todo el ensamblaje que explican en conjunto un $93.5 \%$ de la estructuración de la turbera, repercutiendo en una alta similitud promedio $(81.79 \%)$ para este sector no intervenido (Tabla 1). Por otra parte, en la zona intervenida S. magellanicum presenta una baja contribución (2.33\%), explicado por la disminución del nivel freático generado por el drenaje perimetral hecho para extraer la turba, lo cual se ajusta a lo observado para otras especies del género Sphagnum en el hemisferio norte y en trabajos de restauración (Elisabeth et al. 2007, Robroeket al. 2007). Para el caso de E. rubrum, se observa un incremento de un $42 \%$ en la contribución $(66,03)$ respecto de la zona no intervenida. Esto se debe al aumento de la cobertura de esta especie ya que la misma puede tolerar en forma favorable la disminución del nivel freático generada por la actividad minera. En Tierra del Fuego E. rubrum es considerada una maleza por su escaso valor forrajero (Borrelli \& Oliva, 2001). Además por su capacidad de colonizar suelos ácidos y desnudos en los que no prosperan otras especies, principalmente sobre suelos degradados por sobrepastoreo en coironales en la estepa, en los que se ha eliminado la cubierta herbácea original, según se ha observado en el Parque Nacional Pali Aike y en Tierra del Fuego (Domínguez \& Pauchard, 2012, Collantes et al. 1989).

Otro taxa que caracteriza la zona intervenida con una contribución de 10,41\% es Polytrichum strictum, especie briófita pionera en procesos de sucesión, que crece sobre turba desnuda gracias a que posee un sistema de conducción de agua interno que le permite desarrollarse en ambientes más secos y rizoides con un tegumento que fija la turba desnuda, que le permite retener la humedad en el suelo (Elisabethet al. 2007). Entre las hierbas destacan Holcus lanatus por producir una gran cantidad de semillas y Carex canescens, ambas consideradas plantas forrajeras para la actividad ganadera. La presencia de ellas en la turbera intervenida, representa un claro indicador del impacto en la estructura y composición florística original de este tipo de humedal. Si bien, la mayoría de las especies no nativas en promedio contribuyen individualmente en un $0.3 \%$ (sin considerar a $C$. canescens y $H$. lanatus), en su conjunto aportan un $11.15 \%$ en la formación del ensamblaje, dando cuenta de un riesgo latente como especies invasoras en la turbera intervenida. Al respecto, la presencia de la especie Hieracium pilosella en la turbera intervenida es preocupante debido a que es catalogada en términos agronómicos como una de las malezas más peligrosas a nivel internacional (Krahulcova \& Krahulec, 1999). Su presencia está asociada a pastizales de uso ganadero (Rose et al. 1998). Está incluida en la lista A2 de plagas cuarentenarias desde el año 2000 para Argentina, de acuerdo a la Ley 25.217, mientras que en Chile está 
incluida en el listado de especies exóticas herbáceas más nocivas para cultivos agrícolas (Zilleret al. 2005)

El crecimiento sostenido de la actividad agrícola a nivel global, previsto según estimaciones de la FAO 2011, generará una demanda por la turba y una presión sobre las turberas de Sphagnum en la Región de Magallanes en los próximos años. Si estas proyecciones se confirman se espera que esta actividad industrial deje una importante superficie de turba desnuda durante el proceso de extracción, la cual favorecerá, cambios irreversibles en las funciones de producción y acumulación de turba debido al arribo de especies exóticas invasoras, como las registradas en este estudio. Por otra parte, ya han sido halladas especies invasoras en turberas intervenidas que se encuentran en su fase de operación, esto podría representar un obstáculo para la comercialización de la turba como materia prima de sustratos comerciales, ya que estos no deben presentar diásporas de ninguna especie muchos menos de especies no nativas del tipo invasora. En este sentido, la actividad industrial de turba en Magallanes, deberá evaluar estrategias de manejo destinadas al control de especies exóticas e implementar planes de restauración, para recuperar la cubierta vegetal original.

Finalmente, la modificación mecánica de las turberas como resultado de la extracción de turba y la construcción de canales de drenaje, favorece el arribo de especies exóticas, propias de ambientes más secos como: Rumex acetosella, Holcus lanatus, Carex canescens y Hieracium pilosella. Se recomienda establecer un plan de acción de restauración ecológica antes y durante la etapa de abandono, con el fin de ayudar a restablecer la función de producir y acumular turba que es propia de estos humedales, cerrando los drenajes y realizando las obras necesarias para restablecer el nivel freático característico y la flora original del lugar. Además se recomienda implementar un plan de control de plantas exóticas durante el proceso de extracción de turba, a través de (1) eliminación completa de toda fuente de propágulos y (2) control sistemático mediante la elaboración de mapas de distribución, proporcionando la información básica para planificar la eliminación o contención de especies exóticas, de esta manera se evitará la contaminación de la turba y se facilitará el proceso de restauración.

\section{CONCLUSIONES}

La extracción de turba, genera cambios significativos en la cobertura y la composición de la flora original de una turbera de Sphagnum.

De acuerdo a los datos las plantas exóticas ingresan sólo en sitios intervenidos mecánicamente.

En este sentido, la actividad industrial de turba en Magallanes, deberá evaluar estrategias de manejo destinadas al control de especies exóticas e implementar planes de abandono que contemplen recuperar la cubierta vegetal original y los servicios ecosistémicos que proporcionan estos humedales.

\section{AGRADECIMIENTOS}

El desarrollo de este trabajo fue financiado por el programa: Bases Ambientales, Jurídicas y Comerciales para el Desarrollo Sustentable de las Turberas en Magallanes. Financiado por el Fondo de Desarrollo de Magallanes. Agradecemos también a Juan Larraín por la identificación de briófitas, a Gabriel Quilahuilque por el diseño de la cartografía, y a Rodolfo Iturraspe y Juan Carlos Aravena por el apoyo en terreno y por sus comentarios al manuscrito original y aportes al programa.

\section{LITERATURA CITADA}

Borrelli, P. \& G. Oliva 2001. Producción animal sobre pastizales naturales. Cap. 4. p. 101-130. En Borrelli, P. \& G. Oliva (Eds.): Ganadería sustentable en la Patagonia Austral. Instituto Nacional de Tecnología Agropecuaria (INTA), Centro Regional Patagonia Sur, Río Gallegos, Santa Cruz, Argentina. 270 pp.

Bray J.R. \& J.T. Curtis 1957. An Ordination of the Upland Forest Communities of Southern Wisconsin. Ecological Monographs, 27: 325-349.

Broder, T., C. Blodau, H. Biester \& H. Knorr 2012. Peat descomposition records in three pristine ombrotrophic bogs in southern Patagonia. Biogeosciences, 9:1479-1491.

Caso, C \& M.A. Gil 1988. The gini-simpson index of diversity: estimation in the stratified sampling. Communications in Statistics - Theory and Methods, 17: 2981-2995.

Clarke, K.R. \& R.H. Green 1988. Statistical design and analysis for a 'biological effects' 
study. Marine Ecology Progress Series, 46: 213-226.

Clarke, K.R. 1993. Non-parametric multivariate analyses of change in community structure. Australian Journal of Ecology, 18: 117-143.

Clarke, K.R., P.J. Somerfield \& M.G. Chapman 2006. On resemblance measures for ecological studies, including taxonomic dissimilarities and a zero-adjusted Bray-Curtis coefficient for denuded assemblages. Journal of Experimental Marine Biology and Ecology, 330: 55-80.

Collantes, M., J. Achorena \& G. Koremblit 1989. A soil nutrient gradient in Magallanic Empetrum Heathlands. Vegetatio, 80:183-193.

Díaz, M., J. Larraín, G. Zegers \& C. Tapia 2008. Caracterización florística e hidrológica de turberas de la Isla Grande de Chiloé, Chile. Revista Chilena de Historia Natural, 81: 455-468.

Domínguez, E. \& A. Pauchard 2012. Ten Years of Exclusion From Grazing Increase Native Plant Diversity in the Austral Patagonian Steppe. Cap. V. p 59-81. En: J. R. Arévalo. (Ed.): Grazing Ecology Vegetation \& Soil Impact. Nova Science Publishers Inc. in Hauppauge, N.Y. 195 pp.

Domínguez, E., A. Elvebakk, C. Marticorena \& A. Pauchard 2006. Plantas introducidas en el Parque Nacional Torres del Paine, Chile. Gayana Botánica, 63(2): 131-141.

Elisabeth, V., G. Groeneveld, Ariane Masse' \& L. Rochefort 2007. Polytrichum strictum as a Nurse-Plant in Peatland Restoration. Restoration Ecology, 15(4):709-719.

Espinoza, N. 1996. Malezas presentes en Chile. Instituto de Investigaciones Agropecuarias Carillanca. Concepción. Editora Aníbal Pinto S.A. 219 pp.

FAO. 2011. Perspectivas Alimentarías, Análisis de los mercados mundiales, noviembre de 20112020. OCDE-FAO. 5 pp.

Grootjans, A., R. Iturraspe, A. Lanting, C. Fritz \& H. Joosten 2010. Ecohydrological features of some contrasting mires in Tierra del Fuego, Argentina. Mires \& Peat, 6: 01-15.

Henríquez, J.M. 2004. Estado de la turba esfagnosa en Magallanes 93-106. En: D. Blanco y V. De la Balze (Eds.). Los turbales de la Patagonia. Bases para su inventario y conservación de la biodiversidad. Wetlands International, publicación $\mathrm{N}^{\circ}$. 19, Buenos Aires.

Henríquez, J.M., E. Pisano \& C. Marticorena 1995. Catálogo de la flora vascular de Magallanes (XII Región), Chile. Anales del Instituto de la Patagonia, Serie Ciencias Naturales, 23: 5-30. Iturraspe, R. 2010. Las turberas de Tierra del Fuego y el cambio climático global. Fundación Humedales / Wetlands International. Buenos Aires, Argentina. 26 pp.

IUSS Grupo de trabajo WRB. 2007. Base Referencial Mundial del Recurso Suelo. Primera actualización 2007. Informes sobre Recursos Mundiales de Suelos №. 103. FAO, Roma. 130 pp.

Joosten, H. \& D. Clarke 2002. Wise Use of Mires and Peatlands. International Mire Conservation Group and International Peat Society, Saarijärven Offset Oy, Saarijärvi, Finland. 504 pp.

Joosten, H. \& J. Cowenberg 2008. Peatlands and Carbon. En: Parish, F., A. Sirin, D. Charman, H. Joosten, T.Minayeva, M. Silvius \& L. Stringer (Eds): Assessment on Peatlands, Biodiversity and Climate Change: 99-117. Main Report. Global Env. Centre, K. Lumpur \& Wetlands International, Wageningen.

Kleinebecker, T., N. Holzel \& A. Vogel 2010. Patterns and gradients of diversity in South Patagonian ombrotrophic peat bogs. Austral Ecology, 35: 1-12.

Krahulcova, A. \& F. Krahulec 1999. Chromosome numbers and reproductive systems in selected representatives of Hieraciumsubgen. Pilosella in the KrkonoseMts (the Sudeten Mts). Preslia, 71: 217-234.

Kruskal, J.B. 1964. Nonmetric multidimensional scaling. Psychometrika, 29: 1-27.

Landry J., N. Bahamonde, Garcia Huidobro J., C. Tapia \& L. Rochefort 2010. Canadian peatland restoration framework: A Restoration experience in Chilean peat bogs. Peatlands Interntional, 2: 50-53.

Legendre, L. \& P. Legendre 1983. Numerical Ecology.Developments in Environmental Modelling. Elsevier Scientific publishing Company. The Netherland. 419 pp.

Mack, R. N., D. Simberloff, W. M. Lonsdale, H. Evans, M. Clout \& F. A. Bazzaz 2000. Biotic 
invasions: Causes, epidemiology, global consequences, and control. Ecology Applications, 10: 689-710.

Matthei, O. 1995. Manual de las malezas que crecen en Chile. Alfabeta Impresores, Santiago. $554 \mathrm{pp}$.

Parish, F., A. Sirin, D. Charman, H. Joosten, T. Minayeva, M. Silvius, \& L. Stringer (Eds.). 2008. Assessment on Peatlands, Biodiversity and Climate Change. Main Report. Global Env. Centre y Wetlands International, Wageningen. $179 \mathrm{pp}$.

Pisano, E. 1977. Fitogeografía de Fuego-Patagonia Chilena. I. Comunidades vegetales entre las latitudes $52^{\circ}$ y $56^{\circ} \mathrm{S}$. Anales del Instituto de la Patagonia, 8: 121-250.

Pisano, E. 1983. The Magellanic Complex Tundra. In: AJP Gore (ed.) Mires, swamps, bog, fen and moor, B Regional studies. (Ecosistems of the world 4B). Amsterdam. 295-329 pp.

Pliscoff, P. \& F. Luebert 2006. Ecosistemas terrestres. En: Biodiversidad de Chile: $\mathrm{Pa}$ trimonio y desafíos, pp. 74-87. Comisión Nacional del Medio Ambiente, Santiago. Chile, 639 pp.

Pysek, P., D. Richardson, M. Rejmanek, G. Webster, M. Williamson \& J. Kirschner. 2004. Alien plants in checklist and floras: forwards better communications between taxonomist and ecologists. Taxon, 53(1): 131-143.

Ramsar. 2004. Manuales Ramsar para el uso racional de los humedales. Secretaría de la Convención de Ramsar, Gland (Suiza). Segunda edición. 17 pp.

Robroek, B., Limpens. J., Breeuwer, A. \& M. Schouten 2007. Effects of water level and temperature on performance of four Sphagnum mosses. Plant Ecology, 190: 97-107.

Rochefort, L. 2000. Sphagnum - A keystone genus in habitat restoration. The Bryologist, 103: 503-508.
Roig, C. \& F.A. Roig 2004. Consideraciones generales. 2-21. En: D. Blanco y V. De la Balze (Eds.). Los turbales de la Patagonia. Bases para su inventario y conservación de la biodiversidad. Wetlands International, publicación $\mathrm{N}^{\circ} 19$, Buenos Aires.

Rose, A.B., L.R. Basher, S.K. Wiser, K.H. Platt \& I.H. Lynn 1998. Factors predisposing shorttussock grasslands to Hieracium invasion in Marlborough, New Zealand, 22(2):121-140.

Ruiz, J. \& M. Doberti 2005. Catastro y caracterización de los turbales de Magallanes. Programa Fondema. Código BIP N²0196401-0.123 pp.

Salin, P. 1996. Many uses for peatland cut-away areas. pp. 128-129. In: Vasander. H (ed.). Pealand in Filand, Finnish Peatland Society. Helswki. Finland.

Shannon, C. 1948. A mathematical theory of communication. The Bell System Technical Journal, 27: 379-423.

Teneb, E. \& O. Dollenz 2004. Distribución espacial de la flora vascular, humedad y $\mathrm{pH}$ en un turbal de esfagno (Sphagnum magellanicum Brid.), Magallanes, Chile. Anales del Instituto de la Patagonia, 32: 5-12.

Teneb, E., P. Gómez \& M. González 2008. Observaciones sobre la flora y vegetación de dos turberas en la Región de Aisén, Patagonia Chilena. Gayana Botánica, 65(2): 229-232.

Warwick, R.M. \& K.R. Clarke 1993. Increased variability as a symptom of stress in marine communities. Journal of Experimental Marine Biology and Ecology, 172: 215-226.

Ziller, S.R., J.K Reaser, L.E. Neville \& K. Brandt (eds). 2005. "Invasive alien species in South America" (Especies alienígenas invasoras en Sudamérica): "national reports\& directory of resources" (informes nacionales \& directorio de recursos). "Global Invasive Species Programme, Cape Town, South África". (Programa Global de Especies Invasoras, Ciudad del Cabo, Sudáfrica). 114 pp. 
